\title{
Prospective associations between dietary patterns and body composition changes in European children: the IDEFICS study
}

Juan Miguel Fernández-Alvira ${ }^{1,2, *}$, Karin Bammann ${ }^{3}$, Gabriele Eiben ${ }^{4}$, Antje Hebestreit ${ }^{5}$, Yannis A Kourides ${ }^{6}$, Eva Kovacs ${ }^{7}$, Nathalie Michels ${ }^{8}$, Valeria Pala ${ }^{9}$, Lucia Reisch ${ }^{10}$, Paola Russo ${ }^{11}$, Tomas Veidebaum ${ }^{12}$, Luis A Moreno ${ }^{1,13,14}$ and Claudia Börnhorst ${ }^{5}$ on behalf of the IDEFICS Consortium

${ }^{1}$ GENUD (Growth, Exercise, NUtrition and Development) Research Group, Faculty of Health Sciences, University of Zaragoza, Edificio del SAI, C/Pedro Cerbuna s/n, 50009 Zaragoza, Spain: ${ }^{2}$ Fundación Centro Nacional de Investigaciones Cardiovasculares Carlos III, Madrid, Spain: ${ }^{3}$ Institute for Public Health and Nursing Sciences (IPP), University of Bremen, Bremen, Germany: ${ }^{4}$ Department of Public Health and Community Medicine, Public Health Epidemiology Unit (EPI), Sahlgrenska Academy, University of Gothenburg, Gothenburg, Sweden: ${ }^{5}$ Leibniz Institute for Prevention Research and Epidemiology - BIPS, Bremen, Germany: ${ }^{6}$ Research and Education Institute of Child Health, Strovolos, Cyprus: ${ }^{7}$ Institute for Medical Information Processing, Biometrics and Epidemiology and German Center for Vertigo and Balance Disorders, Ludwig-Maximilians-University, Munich, Germany: ${ }^{8}$ Department of Public Health, Ghent University, Ghent, Belgium: ' Department of Preventive and Predictive Medicine, Epidemiology Unit, Fondazione IRCCS Istituto Nazionale dei Tumori, Milan, Italy: ${ }^{10}$ Copenhagen Business School, Copenhagen, Denmark: ${ }^{11}$ Institute of Food Sciences, National Research Council, Avellino, Italy: ${ }^{12}$ Department of Chronic Diseases, National Institute for Health Development, Tallinn, Estonia: ${ }^{13}$ Instituto Agroalimentario de Aragón (IA2), Instituto de Investigación Sanitaria Aragón (IIS Aragón), Aragón, Spain: ${ }^{14}$ Centro de Investigación Biomédica en Red de Fisiopatología de la Obesidad y Nutrición (CIBERObn), Spain

Submitted 22 February 2017: Final revision received 6 July 2017: Accepted 24 July 2017: First published online 7 September 2017

\begin{abstract}
Objective: To describe dietary patterns by applying cluster analysis and to describe the cluster memberships of European children over time and their association with body composition changes.

Design: The analyses included $k$-means clustering based on the similarities between the relative frequencies of consumption of forty-three food items and regression models were fitted to assess the association between dietary patterns and body composition changes.

Setting: Primary schools and pre-schools of selected regions in Italy, Estonia, Cyprus, Belgium, Sweden, Hungary, Germany and Spain.

Subjects: Participants ( $n$ 8341) in the baseline (2-9 years old) and follow-up (4-11 years old) surveys of the IDEFICS (Identification and prevention of Dietaryand lifestyle-induced health EFfects In Children and infantS) study.

Results: Three persistent clusters were obtained at baseline and follow-up. Children consistently allocated to the 'processed' cluster presented increased BMI $(\beta=0.050 ; 95 \%$ CI $0.006,0.093)$, increased waist circumference $(\beta=0.071 ; 95 \% \mathrm{CI}$ $0 \cdot 001,0 \cdot 141)$ and increased fat mass gain $(\beta=0.052 ; 95 \%$ CI $0.014,0.090)$ over time $v$. children allocated to the 'healthy' cluster. Being in the 'processed'-'sweet' cluster combination was also linked to increased BMI $(\beta=0.079 ; 95 \%$ CI $0.015,0.143)$, increased waist circumference $(\beta=0.172 ; 95 \%$ CI $0.069,0.275)$ and increased fat mass gain $(\beta=0 \cdot 076 ; 95 \%$ CI $0 \cdot 019,0 \cdot 133)$ over time $v$. the 'healthy' cluster.

Conclusions: Children consistently showing a processed dietary pattern or changing from a processed pattern to a sweet pattern presented the most unfavourable changes in fat mass and abdominal fat. These findings support the need to promote overall healthy dietary habits in obesity prevention and health promotion programmes targeting children.
\end{abstract}

Keywords Cluster analysis Dietary patterns Prospective analysis Body composition Children IDEFICS 
Childhood overweight and obesity are associated with high prevalence of a number of co-morbidities and psychosocial disadvantages, representing one of the major public health concerns worldwide ${ }^{(1)}$. While obesity prevalence seems to be levelling off in some countries ${ }^{(2,3)}$, developing countries are experiencing concurrent rapid shifts in diet and activity levels and rising overweight prevalence $^{(4)}$. In addition, population groups with lower education and income levels present a higher obesity burden $^{(5)}$. As childhood overweight and obesity track into adulthood, the increasing prevalence will entail future community health consequences further increasing the risk of developing non-communicable diseases at younger ages $^{(6,7)}$.

The childhood overweight epidemic is largely determined by global changes in lifestyle behaviours leading to an imbalance between energy intake (dietary habits) and energy expenditure (BMR and physical activity). Previous research described the most relevant energy balancerelated behaviours in children and adolescents, showing that among them, poor dietary habits are linked to worse body composition indicators, especially for specific items such as sugared drinks ${ }^{(8)}$ and fast foods ${ }^{(9)}$. However, examining multiple dietary factors in the context of dietary patterns may better explain the risk of developing overweight and obesity than examining single foods or nutrients.

Dietary pattern analysis has been increasingly applied to assess the relationship between overall diet and the risk of chronic diseases, including obesity ${ }^{(10,11)}$. It allows to evaluate the impact of the whole diet, given that foods are consumed in combination. In a previous study, we analysed the prospective associations between socioeconomic status and dietary patterns in the IDEFICS (Identification and prevention of Dietary- and lifestyleinduced health EFfects In Children and infantS) study, applying cluster analysis at two points in time ${ }^{(12)}$. Cluster analysis, one of the most commonly applied methods to derive dietary patterns, clusters individuals into nonoverlapping groups that reflect relatively homogeneous dietary patterns within groups and distinct dietary patterns between groups.

Exploring changes in children's diet over time may help to identify changes in dietary patterns and/or children changing their dietary patterns, thus allowing a better understanding of the impact of diet on body composition. Changes in diet over time and the associations with overweight markers have been previously explored using principal components analysis (PCA) ${ }^{(13-16)}$ and reduced rank regression (RRR $)^{(17,18)}$, but to the best of our knowledge, few studies have examined children's dietary patterns over time using cluster analysis ${ }^{(12,19)}$ and none of them explored the association with changes in body composition. PCA provides linear combinations of foods instead of referring to identifiable groups of individuals. Applying cluster analysis to describe dietary patterns throughout time allows identifying groups of children with consistently healthy/unhealthy diets, providing further insight into dietary impact on body composition changes.

The present study aims to investigate the associations of dietary patterns obtained by applying cluster analysis to children participating in the baseline and follow-up surveys of the IDEFICS study with changes in body composition including BMI, waist circumference (WC), fat mass index (FMI) and fat-free mass index (FFMI).

\section{Participants and methods}

The IDEFICS study is a large multi-centre populationbased study of children aged 2-9 years in selected regions of eight European countries (Belgium, Cyprus, Estonia, Germany, Hungary, Italy, Spain and Sweden) including an embedded intervention ${ }^{(20,21)}$. Two main surveys (baseline (T0) and follow-up (T1)) were conducted in pre-schools and primary school classes (first and second grades at baseline). The baseline survey (September 2007-May 2008) included 16228 children aged 2-9 years (median age $=6.3$ years). The follow-up survey (September 2009May 2010) reached a $68 \%$ overall response and included 11038 children aged 4-11 years. The follow-up survey was synchronized with the baseline to account for seasonal variation. Details of the general design, instruments and survey characteristics can be found elsewhere ${ }^{(20,21)}$. The present study includes only children with less than $50 \%$ of missing values in the food frequency data at baseline and follow-up and for whom complete anthropometric information was available ( $n 8341$ children; $49.9 \%$ boys). Excluded participants presented a slightly higher proportion of overweight/obesity and a higher percentage of lower educated parents (see online supplementary material, Supplemental Table 1). The study was conducted according to the Declaration of Helsinki. Each participating centre obtained ethical approval from its health research ethics authority. All children provided oral consent and their parents provided written informed consent for all examinations and for the collection of samples, subsequent analysis and storage of personal data and collected samples.

\section{Measurements}

\section{Dietary data}

Dietary data were obtained at baseline and follow-up surveys applying the food frequency section of the Children's Eating Habits Questionnaire-Food Frequency Questionnaire (CEHQ-FFQ) ${ }^{(22)}$, a validated screening tool in which the parents report the frequency of the child's consumption of selected food items during the preceding 4 weeks. The questionnaire referred to meals outside the school canteen or childcare meal provision settings only (i.e. under parental control) ${ }^{(22,23)}$. The CEHQ-FFQ includes forty-three food items from food groups and was 
designed as a screening instrument to investigate the consumption of foods shown to be related, either positively or negatively, to overweight and obesity in children, but not to provide an estimate of total energy intake or total food intake ${ }^{(23)}$. Response options displayed from left to right were as follows: 'Never/less than once a week'; '1-3 times a week'; '4-6 times a week'; '1 time per day'; '2 times per day'; ' 3 times per day'; '4 or more times per day'; 'I have no idea'. Only children with $<50 \%$ of missing values were taken into account (92.6\% of participants at baseline and $90.2 \%$ at follow-up). Multiple imputation was applied using sex, age, BMI and country as predictors for the remaining missing values.

\section{Anthropometric measurements}

Although BMI has been widely reported as an indicator of overweight and obesity due to its simplicity, it is not a perfect indicator of excess body fat ${ }^{(24,25)}$. Therefore, other body composition indicators, such as WC for assessing abdominal fat, and FMI and FFMI as indicators of overall fat/lean tissue in relation to total mass, might help to complement BMI when assessing dietary impact on body composition. Trained staff carried out anthropometric measurements at baseline and follow-up following a standardized procedure. Body height was measured without shoes and all plaits undone using a portable stadiometer (model: telescopic height measuring instrument SECA 225) to the nearest $0 \cdot 1 \mathrm{~cm}$. Weight was measured to the nearest $0 \cdot 1 \mathrm{~kg}$ by means of a child-adapted version of the electronic scale Tanita BC 420 SMA, with the children in fasting status (more than $8 \mathrm{~h}$ since last meal) and wearing only underwear ${ }^{(26)}$. For each child, BMI, BMI categories and $Z$-scores of BMI and of BMI change over the 2-year follow-up were determined according to age- and genderspecific BMI reference standards according to the criteria proposed by the International Obesity Task Force ${ }^{(27)}$.

WC was measured using a non-elastic tape (Seca 200) at the midpoint between the iliac crest and the lower border of the tenth rib in a standing position to the nearest $0.1 \mathrm{~cm}$. For each child, $Z$-scores of WC and of WC change over the 2-year follow up were also determined according to ageand gender specific reference standards of the International Obesity Task Force ${ }^{(27)}$.

Skinfold measurements were taken on the right side of the body at the triceps (halfway between the acromion and the olecranon process at the back of the arm) and the subscapular site (about $2 \mathrm{~cm}$ below the tip of the scapula at an angle of $45^{\circ}$ to the lateral side of the body) with a skinfold calliper (Holtain, range $0-40 \mathrm{~mm}$ ) to the nearest $0.2 \mathrm{~mm}$. For each child, body fat mass was calculated using the gender-specific Slaughter's equations. FMI was calculated by dividing body fat mass in kilograms by the square of height in metres. FFMI was calculated by dividing lean mass in kilograms by the square of height in metres. Finally, FMI and FFMI age- and gender-specific $Z$-scores were derived.

\section{Parental questionnaire variables}

During the baseline and follow-up surveys, parents completed a self-administered questionnaire on parental attitudes, children's behaviour and social environment. Parental education and income were self-reported. Parental education level was categorized according to the International Standard Classification of Education (ISCED-97) ${ }^{(28)}$. Household income was assessed using nine country-specific categories based on the median equivalent income. The gained amount was then equalized to the number of household members using the Organisation for Economic Co-operation and Development's square root scale ${ }^{(29)}$. Additionally, a migrant background was assumed if one or both of the parents were born in another country. For the purposes of the present analysis, an additive socio-economic status indicator constructed by Bammann et al. ${ }^{(30)}$ was used.

To assess physical activity, parents reported how many hours and minutes their child spent playing outdoors on a typical weekday and weekend day during the previous month. In addition, parents reported how many hours and minutes per week the child spent doing sport in a sports club. Finally, total physical activity time was calculated and expressed as $\mathrm{h} /$ week.

\section{Statistical methods}

In a previous IDEFICS report, $k$-means cluster analysis was performed to identify clusters of children with similar dietary patterns at baseline and follow-up. The complete procedure and the derived dietary patterns have been described in a previous paper ${ }^{(12)}$ and are taken into account for the purposes of the present study. Briefly, the authors described three persistent dietary patterns in children (see online supplementary material, Supplemental Tables 2 and 3): (i) 'processed' (higher frequency of takeaway and high-fat foods, such as savoury pastries, fritters, pizza as main dish, fried potatoes, hamburger, hot dog, kebab and wraps, and lowest scores for products such as wholemeal bread, cooked vegetables, raw vegetables, and fresh fruits without added sugar); (ii) 'sweet' (higher frequency of consumption of sugar-rich products, like chocolate- or nut-based spread, sweetened drinks, fruit juices, diet drinks, candies, loose candies, marshmallows, and biscuits, packaged cakes, pastries and puddings, with the lowest scores for water, porridge, oatmeal, gruel, unsweetened cereals, muesli, raw vegetables, plain unsweetened milk and plain unsweetened yoghurt); and (iii) 'healthy' (higher frequency of consumption of low-fat foods, foods rich in vitamins and wholegrain foods, e.g. raw vegetables, fresh fruits without added sugar, porridge, oatmeal, gruel, unsweetened cereals, muesli and plain unsweetened milk, and the lowest values for high-fat, high-sugar products, such as fried potatoes, sweetened drinks, sweetened milk, mayonnaise and mayonnaise-based products, chocolateor nut-based spread, crisps, corn crisps, popcorn, and 
biscuits, packaged cakes, pastries and puddings). To assess the changes in dietary patterns over time, children's cluster memberships at baseline and follow-up were cross-tabulated, showing the proportion of children being allocated to the same or different clusters.

The associations of body composition changes with dietary patterns at baseline (T0) and follow-up (T1) were estimated based on mixed models with random effects for country and setting (school) to account for the clustered study design. Dietary patterns at baseline and follow-up were the predictor variables and gender, age, intervention $v$. control, socioeconomic status score, physical activity and baseline predictor levels were the covariates. Analogous models were applied for estimating body composition changes according to different dietary pattern combinations (i.e. 'healthy', 'sweet' or 'processed' at both times; 'processed' at T0 and 'healthy' at T1 ('processed'-'healthy'); 'sweet' at T0 and 'processed' at T1 ('sweet'-'processed'); etc.). The statistical significance level was set at $P \leq 0 \cdot 05$. The analyses were performed using the statistical software package IBM SPSS Statistics version 20.0 .

\section{Results}

Table 1 shows the characteristics of the study sample. Among the total sample, $6.5 \%$ was classified as obese and $12.3 \%$ as overweight, while $10.4 \%$ was classified as underweight. Based on forty-two food items and their relative frequency of consumption, the most prevalent dietary pattern was the 'processed' cluster at baseline ( $n 4076$ at T0, $n 2360$ at T1) while the 'healthy' cluster was the most prevalent at follow-up ( $n 2558$ at T0, $n 4256$ at T1). The 'sweet' cluster showed similar numbers at baseline and follow-up ( $n 1707$ at T0, $n 1725$ at T1). Regarding gender differences, the 'healthy' pattern was significantly more prevalent in girls compared with boys. Table 2 presents the mean values of body composition indicators and their standard deviations in the three clusters at baseline and follow-up. The highest BMI $Z$-score and WC $Z$-score means were found in the 'processed' cluster both at baseline and follow-up. Likewise, the highest FFMI $Z$-score and FMI $Z$-score means were found in the 'processed' cluster. The lowest values in all four indicators were found in the 'sweet' cluster.

Table 3 shows the associations of body composition changes at follow-up with dietary patterns at baseline and follow-up. Taking the 'healthy' cluster as reference, being allocated to the 'processed' cluster at baseline was associated with larger WC $Z$-score change $(\beta=0.079 ; 95 \%$ CI $0.022,0.135)$ at follow-up. Being allocated to the 'processed' cluster at follow-up was also associated with larger BMI $Z$-score change ( $\beta=0 \cdot 039 ; 95 \%$ CI 0.006, 0.071) and larger FMI $Z$-score change $(\beta=0.045 ; 95 \%$ CI $0 \cdot 016$, $0 \cdot 073)$ at follow-up. Taking again the 'healthy' cluster as reference, being allocated to the 'sweet' cluster at baseline was associated with larger WC $Z$-score gain $(\beta=0.078$; $95 \%$ CI 0.006, 0.151) at follow-up, while being allocated to the 'sweet' cluster at follow-up was associated with larger BMI $Z$-score gain $(\beta=0.053 ; 95 \%$ CI $0.014,0.091)$, larger WC $Z$-score gain $(\beta=0.098 ; 95 \%$ CI $0.034,0.161)$ and larger FMI $Z$-score gain $(\beta=0 \cdot 041 ; 95 \%$ CI $0.006,0 \cdot 075)$ at follow-up.

Table 4 and Fig. 1 display the estimated associations between body composition changes and the different prospective cluster combinations. Taking the 'healthy''healthy' combination as reference, children allocated to the 'processed'-'processed' combination presented a larger increase in BMI $Z$-score $(\beta=0.050 ; 95 \%$ CI 0.006 , $0.093)$, larger WC $Z$-score gain $(\beta=0.071 ; 95 \%$ CI 0.001 , $0 \cdot 141)$ and larger FMI $Z$-score increase $(\beta=0.052 ; 95 \% \mathrm{CI}$ $0 \cdot 014,0 \cdot 090)$ at follow-up. Being allocated to the "processed'-'sweet' combination was also associated with larger BMI $Z$-score increase ( $\beta=0 \cdot 079 ; 95 \%$ CI 0.015, 0.143), larger WC $Z$-score increase $(\beta=0 \cdot 172 ; 95 \%$ CI 0.069 , $0 \cdot 275)$ and larger FMI $Z$-score gain $(\beta=0.076 ; 95 \%$ CI $0 \cdot 019,0 \cdot 133)$ at follow-up. Finally, children allocated to the 'sweet'-'sweet' combination presented also a larger WC $Z$-score gain ( $\beta=0 \cdot 127 ; 95 \%$ CI $0 \cdot 038,0 \cdot 216)$ at follow-up.

\section{Discussion}

The present study describes associations between dietary patterns and body composition changes in European children applying cross-sectional and prospective models. A dietary pattern characterized by higher frequency of consumption of snacks and fast foods ('processed') was associated with increased BMI, increased WC and fat mass gain compared with a dietary pattern rich in fruits, vegetables and wholemeal products ('healthy'). The 'sweet' pattern (higher frequency of consumption of sweet foods and sweetened drinks) was also linked to increased BMI, WC and fat mass gain compared with the 'healthy' pattern. All the described associations were observed accounting for country, setting (school), gender, age, intervention $v$. control groups, socio-economic status score, physical activity level and baseline predictor levels.

The current paper is based on dietary patterns derived by cluster analysis at two different points in time in 2- to 9year-old children participating in the IDEFICS study. These patterns presented similar profiles of relative frequencies of food consumption at each point in time, allowing us to describe changes in body composition in those children consistently presenting unhealthier dietary patterns and to compare their body composition changes with the changes in children consistently showing healthier dietary profiles. Among all combinations, the persistent 'processed' pattern (children allocated to the 'processed' cluster at baseline and follow-up) and the 'processed'-'sweet' pattern showed the largest differences in 
Table 1 Characteristics of the study sample: children ( $n$ 8341) aged 2-9 years from eight European countries, baseline survey of the IDEFICS (Identification and prevention of Dietary- and lifestyle-induced health EFfects In Children and infantS) study, September 2007-May 2008

\begin{tabular}{|c|c|c|c|c|c|c|c|}
\hline & \multicolumn{2}{|c|}{ Total } & \multicolumn{2}{|c|}{ Boys ( $n$ 4165) } & \multicolumn{2}{|c|}{ Girls (n 4176) } & \multirow[b]{2}{*}{$P$ value } \\
\hline & $n$ & $\%$ & $n$ & $\%$ & $n$ & $\%$ & \\
\hline \multicolumn{8}{|l|}{ BMI category } \\
\hline Underweight & 865 & $10 \cdot 4$ & 433 & $10 \cdot 4$ & 432 & $10 \cdot 4$ & 0.143 \\
\hline Normal weight & 5906 & $70 \cdot 8$ & 3019 & $72 \cdot 5$ & 2887 & $69 \cdot 1$ & \\
\hline Overweight & 1025 & $12 \cdot 3$ & 445 & $10 \cdot 7$ & 580 & 13.9 & \\
\hline Obesity & 545 & 6.5 & 268 & $6 \cdot 4$ & 277 & $6 \cdot 6$ & \\
\hline \multicolumn{8}{|l|}{ Country } \\
\hline Italy & 1352 & $16 \cdot 2$ & 690 & $16 \cdot 6$ & 662 & $15 \cdot 9$ & 0.876 \\
\hline Estonia & 1063 & $12 \cdot 7$ & 511 & $12 \cdot 3$ & 552 & $13 \cdot 2$ & \\
\hline Cyprus & 962 & 11.5 & 503 & $12 \cdot 1$ & 459 & 11.0 & \\
\hline Belgium & 931 & 11.2 & 469 & $11 \cdot 3$ & 462 & $11 \cdot 1$ & \\
\hline Sweden & 1110 & $13 \cdot 3$ & 530 & $12 \cdot 7$ & 580 & $13 \cdot 9$ & \\
\hline Germany & 915 & 11.0 & 457 & 11.0 & 458 & $11 \cdot 0$ & \\
\hline Hungary & 950 & 11.4 & 472 & $11 \cdot 3$ & 478 & 11.4 & \\
\hline Spain & 1058 & $12 \cdot 7$ & 533 & $12 \cdot 8$ & 525 & $12 \cdot 6$ & \\
\hline \multicolumn{8}{|c|}{ Family socio-economic status } \\
\hline Low & 290 & 3.5 & 140 & 3.4 & 150 & 3.6 & 0.819 \\
\hline Medium & 3199 & 38.4 & 1620 & 38.9 & 1579 & 37.8 & \\
\hline High & 3747 & 44.9 & 1858 & 44.6 & 1889 & $45 \cdot 2$ & \\
\hline \multicolumn{8}{|c|}{ Dietary pattern at baseline (T0) } \\
\hline 'Processed' & 4076 & 48.9 & 2074 & 49.8 & 2002 & 47.9 & 0.045 \\
\hline 'Sweet' & 1707 & 20.5 & 886 & $21 \cdot 3$ & 821 & $19 \cdot 7$ & \\
\hline 'Healthy' & 2558 & $30 \cdot 7$ & 1205 & 28.9 & 1353 & 32.4 & \\
\hline \multicolumn{8}{|c|}{ Dietary pattern at follow-up (T1) } \\
\hline 'Processed' & 2360 & $28 \cdot 3$ & 1239 & 29.7 & 1121 & $26 \cdot 8$ & $<0.001$ \\
\hline 'Sweet' & 1725 & 20.7 & 914 & 21.9 & 811 & $19 \cdot 4$ & \\
\hline \multirow[t]{2}{*}{ 'Healthy' } & 4256 & $51 \cdot 0$ & 2012 & $48 \cdot 3$ & 2244 & $53 \cdot 7$ & \\
\hline & Mean & SD & Mean & SD & Mean & SD & \\
\hline Age (years) & $6 \cdot 15$ & 1.68 & $6 \cdot 10$ & 1.67 & $6 \cdot 20$ & 1.68 & 0.647 \\
\hline Physical activity (h/week) & $17 \cdot 66$ & $10 \cdot 62$ & $17 \cdot 87$ & $10 \cdot 74$ & $17 \cdot 46$ & $10 \cdot 50$ & 0.039 \\
\hline
\end{tabular}

$P$ value from $X^{2}$ test and Student $t$ test.

Table 2 Body composition indicators by dietary patterns at baseline (T0, September 2007-May 2008) and follow-up (T1, September 2009-May 2010) among children ( $n$ 8341) aged 2-9 years from eight European countries, IDEFICS (Identification and prevention of Dietary- and lifestyle-induced health EFfects In Children and infantS) Study

\begin{tabular}{|c|c|c|c|c|c|c|c|}
\hline Baseline (T0) & Mean & SD & $P$ value & Follow-up (T1) & Mean & SD & $P$ value \\
\hline BMI Z-score T0 & & & & BMI Z-score T1 & & & \\
\hline 'Processed' ( $n$ 4076) & 0.457 & 1.197 & $<0.001$ & 'Processed' (n 2360) & 0.566 & 1.361 & $<0.001$ \\
\hline 'Sweet' $(n$ 1707) & 0.125 & 1.070 & & 'Sweet' ( $n$ 1725) & 0.175 & 1.275 & \\
\hline 'Healthy' ( $n$ 2558) & 0.269 & 1.105 & & 'Healthy' ( $n$ 4256) & 0.456 & 1.331 & \\
\hline WC Z-score T0 & & & & WC Z-score T1 & & & \\
\hline 'Processed' ( $n$ 4076) & 0.383 & 1.529 & $<0.001$ & 'Processed' (n 2360) & 0.704 & 1.348 & $<0.001$ \\
\hline 'Sweet' ( $n$ 1707) & -0.049 & 1.255 & & 'Sweet' ( $n$ 1725) & 0.409 & 1.231 & \\
\hline 'Healthy' (n 2558) & 0.187 & 1.301 & & 'Healthy' (n 4256) & 0.553 & 1.337 & \\
\hline FFMI Z-score T0 & & & & FFMI Z-score T1 & & & \\
\hline 'Processed' (n 4076) & 0.061 & 1.024 & $<0.001$ & 'Processed' (n 2360) & 0.597 & 1.027 & $<0.001$ \\
\hline 'Sweet' ( $n$ 1707) & -0.154 & 0.973 & & 'Sweet' ( $n$ 1725) & -0.174 & 0.946 & \\
\hline 'Healthy' (n 2558) & 0.008 & 0.970 & & 'Healthy' (n 4256) & 0.038 & 0.999 & \\
\hline FMI Z-score T0 & & & & FMI Z-score T1 & & & \\
\hline 'Processed' (n 4076) & 0.097 & 1.067 & $<0.001$ & 'Processed' (n 2360) & 0.089 & 1.062 & $<0.001$ \\
\hline 'Sweet' ( $n$ 1707) & -0.169 & 0.872 & & 'Sweet' ( $n$ 1725) & -0.169 & 0.910 & \\
\hline 'Healthy' ( $n$ 2558) & -0.038 & 0.954 & & 'Healthy' ( $n$ 4256) & 0.021 & 0.993 & \\
\hline
\end{tabular}

WC, waist circumference; FFMI, fat-free mass index; FMI, fat mass index.

$P$ value from ANOVA.

body composition compared with the persistent 'healthy' pattern, with larger increases in body mass and fat gain indicators.

Interestingly, the cross-sectional models at baseline and follow-up showed lower mean BMI, WC and FMI $Z$-score values and higher mean FFMI $Z$-score for the 'sweet' cluster compared with the 'healthy' cluster, suggesting that children with more favourable body composition indicators were more likely to report higher consumption of sugar-rich products. However, the prospective 
Table 3 Body composition change estimates by dietary patterns at baseline (T0, September 2007-May 2008) and follow-up (T1, September 2009-May 2010) among children ( $n$ 8341) aged 2-9 years from eight European countries, IDEFICS (Identification and prevention of Dietary- and lifestyle-induced health EFfects In Children and infantS) Study

\begin{tabular}{|c|c|c|c|c|}
\hline Parameter & Estimate & $95 \% \mathrm{Cl}$ & $t$ & $P$ value \\
\hline \multicolumn{5}{|l|}{ BMI Z-score (IOTF) change } \\
\hline 'Processed' T0 (n 4076) & 0.027 & $-0.008,0.061$ & 1.506 & 0.132 \\
\hline 'Sweet' TO ( $n$ 1707) & 0.033 & $-0.011,0.077$ & 1.453 & 0.147 \\
\hline ‘Healthy' TO ( $n$ 2558) & Ref. & & & \\
\hline 'Processed' T1 (n 2360) & 0.039 & $0.006,0.071$ & $2 \cdot 344$ & 0.019 \\
\hline 'Sweet' T1 ( $n$ 1725) & 0.053 & $0.014,0.091$ & 2.657 & 0.008 \\
\hline 'Healthy' T1 ( $n$ 4256) & Ref. & & & \\
\hline \multicolumn{5}{|l|}{ WC Z-score (IOTF) change } \\
\hline 'Processed' TO ( $n$ 4076) & 0.079 & $0.022,0.135$ & $2 \cdot 737$ & 0.006 \\
\hline 'Sweet' T0 ( $n$ 1707) & 0.078 & $0.006,0.151$ & $2 \cdot 116$ & 0.034 \\
\hline ‘Healthy' TO (n 2558) & Ref. & & & \\
\hline 'Processed' T1 (n 2360) & 0.019 & $-0.034,0.071$ & $0 \cdot 707$ & 0.480 \\
\hline 'Sweet' T1 ( $n$ 1725) & 0.098 & $0.034,0.161$ & 3.022 & 0.003 \\
\hline 'Healthy' T1 (n 4256) & Ref. & & & \\
\hline \multicolumn{5}{|l|}{ FMI Z-score change } \\
\hline 'Processed' TO (n 4076) & 0.026 & $-0.004,0.057$ & 1.649 & 0.099 \\
\hline 'Sweet' T0 ( $n$ 1707) & 0.019 & $-0.021,0.058$ & 0.921 & 0.357 \\
\hline ‘Healthy' TO (n 2558) & Ref. & & & \\
\hline 'Processed' T1 (n 2360) & 0.045 & $0.016,0.073$ & 3.025 & 0.002 \\
\hline 'Sweet' T1 ( $n$ 1725) & 0.041 & $0.006,0.075$ & $2 \cdot 322$ & 0.020 \\
\hline 'Healthy' T1 ( $n$ 4256) & Ref. & & & \\
\hline \multicolumn{5}{|l|}{ FFMI Z-score change } \\
\hline 'Processed' TO (n 4076) & -0.004 & $-0.065,0.056$ & -0.134 & 0.893 \\
\hline 'Sweet' TO ( $n$ 1707) & 0.013 & $-0.066,0.091$ & 0.316 & 0.752 \\
\hline ‘Healthy' TO ( $n$ 2558) & Ref. & & & \\
\hline 'Processed' T1 (n 2360) & -0.048 & $-0.105,0.009$ & $-1 \cdot 641$ & 0.101 \\
\hline 'Sweet' T1 ( $n$ 1725) & -0.014 & $-0.083,0.054$ & -0.405 & 0.685 \\
\hline 'Healthy' T1 ( $n$ 4256) & Ref. & & & \\
\hline
\end{tabular}

IOTF, International Obesity Task Force; WC, waist circumference; FFMI, fat-free mass index; FMI, fat mass index; Ref., reference category.

Mixed models with body composition parameters as the outcome with random effects for country and setting, dietary pattern at T0, T1 or both as predictor variables, and gender, age, intervention $v$. control, socio-economic status score, physical activity level and baseline predictor levels as covariates.

associations showed that body composition changes were more unfavourable for children allocated to the 'sweet' cluster over time (or a combination of 'processed'-'sweet' clustering) compared with children allocated to the 'healthy' cluster over time, regardless of their initial body composition. These results suggest that associations reported in the cross-sectional models may reflect reverse causation, while prospective models might better reflect the true aetiological associations.

Even if dietary patterns are dependent on the population studied and/or the technique applied (cluster analysis, PCA, RRR) and thus not fully comparable between cohorts, previous studies found similar results. Briefly, several reports described 'processed' and 'healthy' dietary patterns in children across different studies. Moreover, some studies explored diet longitudinally and found evidence of tracking of the dietary patterns over time, especially the 'healthy' and 'processed' patterns ${ }^{(19,31-33)}$.

Concerning the associations of dietary patterns and later body composition, on the one hand, some studies found associations between 'energy-dense, high-fat, high-sugar' or 'snacking' dietary patterns and increased $\mathrm{BMI}^{(15)}$ and adiposity measured using $\mathrm{FMI}^{(17,18)}$ over time. On the other hand, a 'health-aware' pattern at age 10 years, characterized by higher consumption of fruits and vegetables and wholegrain products and lower consumption of processed foods and soft drinks, was linked to lower fat mass at age 11 years $^{(34)}$. In Norway, a 'varied Norwegian' pattern rich in vegetables, fruits, unrefined cereal products and fish was related to lower risk of remaining overweight over time from middle childhood ( 9 to 10 years old) to early adolescence (12 to 13 years old) ${ }^{(16)}$.

The results of the present study add value to the main conclusion of a recent review by Ambrosini ${ }^{(11)}$, where the balance of evidence shows that a posteriori dietary patterns defined as high in energy-dense, high-fat and low-fibre foods predispose children and adolescents to later overweight and obesity. Our results are in line with previous findings ${ }^{(13-18)}$ applying an alternative technique (cluster analyses $v$. PCA/RRR) and taking several body composition indices into account.

Unlike PCA, cluster analysis assigns participants to mutually exclusive clusters and does not create continuous dietary pattern $Z$-scores. One advantage of the latter is the ability to track children and their classification over time if equivalent clusters can be derived, while one of the main limitations is the lack of a continuous indicator quantifying the degree to which the individuals' dietary data reflect 
Table 4 Body composition change estimates by prospective dietary pattern combinations at baseline (T0, September 2007-May 2008) and follow-up (T1, September 2009-May 2010) among children ( $n$ 8341) aged 2-9 years from eight European countries, IDEFICS (Identification and prevention of Dietary- and lifestyle-induced health EFfects In Children and infantS) Study

\begin{tabular}{|c|c|c|c|c|}
\hline Parameter & Estimate & $95 \% \mathrm{Cl}$ & $t$ & $P$ value \\
\hline \multicolumn{5}{|l|}{ BMI Z-score (IOTF) change } \\
\hline 'Processed'-'processed' (n 1901) & 0.050 & $0.006,0.093$ & $2 \cdot 255$ & 0.024 \\
\hline 'Sweet'-'processed' (n 198) & 0.015 & $-0.075,0.105$ & 0.330 & 0.741 \\
\hline 'Healthy'-'processed' ( $n$ 261) & 0.020 & $-0.062,0.102$ & 0.483 & 0.629 \\
\hline ‘Processed'-'sweet’ ( $n$ 427) & 0.079 & $0.015,0.143$ & $2 \cdot 413$ & 0.016 \\
\hline 'Sweet'-'sweet' ( $n$ 1159) & 0.050 & $-0.004,0.103$ & 1.822 & 0.069 \\
\hline 'Healthy'-'sweet' ( $n$ 139) & 0.040 & $-0.058,0.139$ & 0.805 & 0.421 \\
\hline 'Processed'-'healthy' (n 1748) & 0.001 & $-0.042,0.043$ & 0.032 & 0.975 \\
\hline 'Sweet'-'healthy' ( $n$ 350) & 0.039 & $-0.030,0.109$ & $1 \cdot 109$ & 0.268 \\
\hline 'Healthy'-'healthy' ( $n$ 2158) & Ref. & & & \\
\hline \multicolumn{5}{|l|}{ WC Z-score (IOTF) change } \\
\hline 'Processed'-'processed' (n 1901) & 0.071 & $0.001,0.141$ & 1.999 & 0.046 \\
\hline 'Sweet'-'processed' (n 198) & -0.015 & $-0.159,0.129$ & -0.200 & 0.842 \\
\hline 'Healthy'-'processed' (n 261) & 0.019 & $-0.113,0.151$ & 0.287 & 0.774 \\
\hline 'Processed'-'sweet' ( $n$ 427) & 0.172 & $0.069,0.275$ & $3 \cdot 268$ & 0.001 \\
\hline 'Sweet'-'sweet' ( $n$ 1159) & 0.127 & $0.038,0.216$ & $2 \cdot 808$ & 0.005 \\
\hline 'Healthy'-'sweet' ( $n$ 139) & 0.000 & $-0 \cdot 158,0.158$ & 0.003 & 0.998 \\
\hline 'Processed'-'healthy' ( $n$ 1748) & 0.058 & $-0.011,0.126$ & 1.644 & $0 \cdot 100$ \\
\hline 'Sweet'-'healthy' ( $n$ 350) & 0.067 & $-0.045,0.179$ & $1 \cdot 166$ & 0.244 \\
\hline 'Healthy'-‘healthy' ( $n$ 2158) & Ref. & & & \\
\hline \multicolumn{5}{|l|}{ FMI Z-score change } \\
\hline 'Processed'-'processed' (n 1901) & 0.052 & $0.014,0.090$ & $2 \cdot 681$ & 0.007 \\
\hline 'Sweet'-'processed' (n 198) & 0.020 & $-0.058,0.099$ & 0.507 & 0.612 \\
\hline 'Healthy'-'processed' (n 261) & 0.040 & $-0.033,0.113$ & 1.079 & 0.281 \\
\hline 'Processed'-'sweet' ( $n$ 427)' & 0.076 & $0.019,0.133$ & 2.626 & 0.009 \\
\hline ‘Sweet'-'sweet' ( $n$ 1159) & 0.030 & $-0.017,0.078$ & 1.246 & 0.213 \\
\hline ‘Healthy'-'sweet' ( $n$ 139) & 0.007 & $-0.077,0.092$ & 0.171 & 0.864 \\
\hline 'Processed'-'healthy' ( $n$ 1748) & 0.001 & $-0.037,0.038$ & 0.023 & 0.982 \\
\hline 'Sweet'-'healthy' ( $n$ 350) & 0.019 & $-0.004,0.081$ & 0.615 & 0.539 \\
\hline 'Healthy'-'healthy' ( $n$ 2158) & Ref. & & & \\
\hline \multicolumn{5}{|l|}{ FFMI Z-score change } \\
\hline 'Processed'-'processed’ (n 1901) & -0.043 & $-0.119,0.032$ & $-1 \cdot 122$ & 0.262 \\
\hline 'Sweet'-'processed' (n 198) & -0.030 & $-0.184,0.125$ & -0.375 & 0.707 \\
\hline 'Healthy'-'processed' ( $n$ 261) & -0.038 & $-0.181,0.105$ & -0.522 & 0.601 \\
\hline 'Processed'-'sweet' ( $n$ 427) & -0.011 & $-0.123,0.100$ & -0.202 & 0.840 \\
\hline ‘Sweet'-'sweet' ( $n$ 1159) & 0.007 & $-0.089,0.104$ & 0.149 & 0.882 \\
\hline 'Healthy'-'sweet' ( $n$ 139) & -0.073 & $-0.240,0.093$ & -0.869 & 0.385 \\
\hline 'Processed'-'healthy' ( $n$ 1748) & 0.011 & $-0.064,0.085$ & 0.279 & 0.780 \\
\hline 'Sweet'-'healthy' ( $n$ 350) & 0.006 & $-0.115,0.128$ & $0 \cdot 101$ & 0.919 \\
\hline 'Healthy'-'healthy' (n 2158) & Ref. & & & \\
\hline
\end{tabular}

IOTF, International Obesity Task Force; WC, waist circumference; FFMI, fat-free mass index; FMI, fat mass index; Ref., reference category Mixed models with body composition parameters as the outcome with random effects for country and setting, dietary pattern at T0, T1 or both as predictor variables, and gender, age, intervention $v$. control, socio-economic status score, physical activity level and baseline predictor levels as covariates.

the described dietary patterns. A common limitation to both methods is their exploratory nature, relying on data covariance and thus dependent on the study sample. Another limitation is that dietary patterns do not provide information about meeting food/nutrient intake recommendations as such. A third method, RRR, combining a priori and a posteriori information may provide a better prediction of the outcome than PCA and cluster analysis, relying to a lesser extent on the study population and thus allowing greater replication of the associations.

The present study is subject to a number of limitations. First, the IDEFICS study was not designed to be representative at national level. Participation in the study was voluntary, thus some population groups, e.g. lower educated families or individuals presenting worse body composition, may have been less willing to take part in the study ${ }^{(35)}$. As no systematic information on non-participants was available, the direction of a possible bias cannot be predicted. In addition, $43 \%$ of the initial baseline cohort did not participate at follow-up and/or did not provide complete data. Excluded participants showed a higher prevalence of overweight/obesity and a higher proportion of lower educated parents (see Supplemental Table 2). Consequently, a selection bias cannot be ruled out. Concerning the dietary information, the CEHQ-FFQ was designed for capturing information on parentsupervised meals but not for recording total food intake. Nevertheless, the data allowed us to describe prospective body composition differences in relation to dietary patterns, as the main inter-individual variation in dietary patterns takes place in the family setting, rather than at school. 


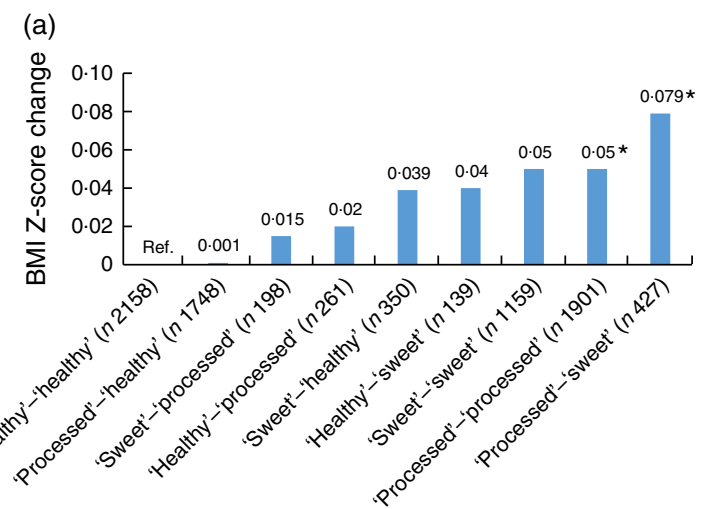

(c)

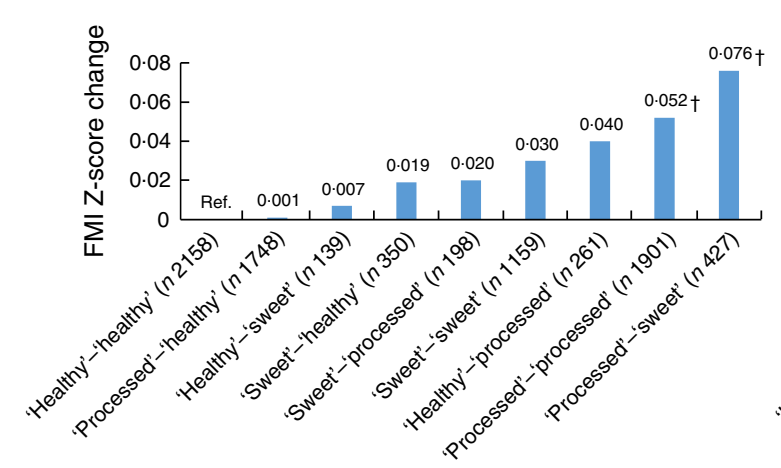

(b)

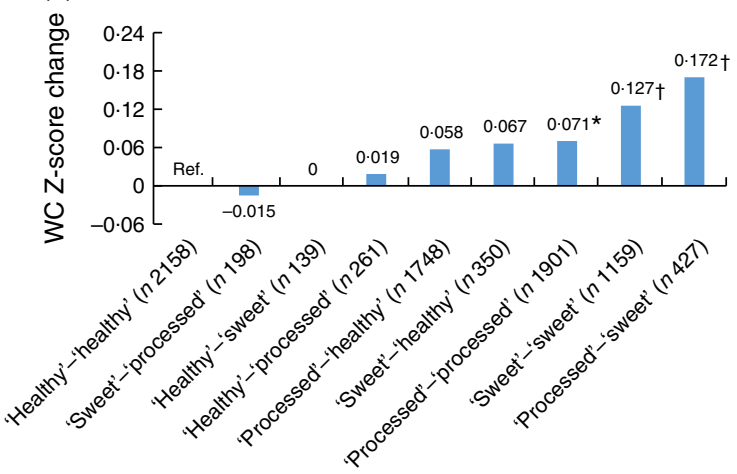

(d)

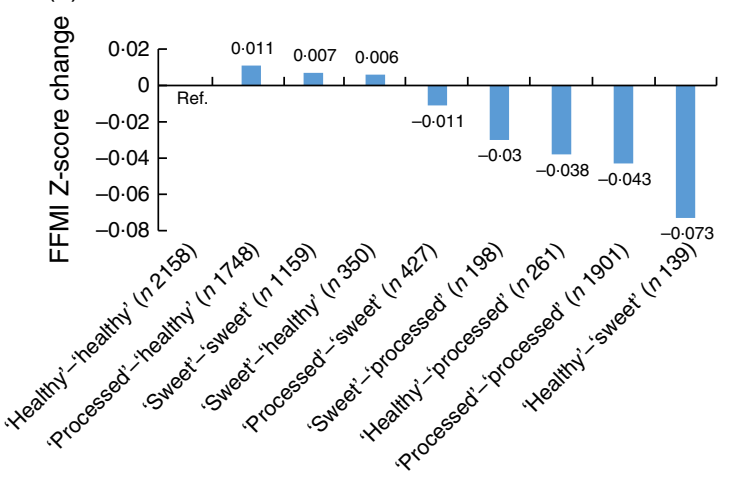

Fig. 1 (a) BMI Z-score change estimates, (b) waist circumference (WC) Z-score change estimates, (c) fat mass index (FMI) Z-score change estimates and (d) fat-free mass index (FFMI) Z-score change estimates by prospective dietary pattern combinations among children ( $n$ 8341) aged 2-9 years at baseline (T0, September 2007-May 2008) and 4-11 years at follow-up (T1, September 2009May 2010) from eight European countries, IDEFICS (Identification and prevention of Dietary- and lifestyle-induced health EFfects In Children and infantS) study. Mixed models with body composition parameters as the outcome, dietary pattern combinations at T0 and $\mathrm{T} 1$ as predictor variables, random effects for country and setting, and gender, age, intervention $v$. control, socio-economic status score, physical activity level and baseline predictor levels as covariates. ${ }^{\star} P<0.05 ; \dagger P<0.001$; Ref., reference category

To the best of our knowledge, the present report is the first on a multi-centre European study assessing dietary patterns over time in relation to prospective body composition changes in children. Another strength is the large sample size, including a wide variety of dietary habits and cultural backgrounds across eight European countries. The use of a validated dietary instrument shown to provide reproducible estimates of consumption frequencies is also one of the main strengths of the study. Deriving dietary patterns by applying cluster analyses at two points in time allowed us to identify children with consistently healthier or unhealthier dietary profiles and to relate them to prospective body composition changes.

Children consistently showing a 'processed' dietary pattern or changing from a 'processed' pattern to a 'sweet' pattern presented the most unfavourable changes in fat mass and abdominal fat. These results support the need of promoting overall healthier dietary patterns in children to prevent overweight and obesity later in life. Examining dietary patterns as a whole may facilitate the explanation of overweight development compared with individual foods or nutrients. Prospective studies provide a clearer picture of the true associations than cross-sectional designs, and therefore more prospective studies are needed to evaluate the generalizability of these results. The use of a combination of techniques, including a priori data-driven techniques (RRR), would allow us to confirm the current findings and to better define public health recommendations.

\section{Acknowledgements}

Acknowledgements: The authors gratefully acknowledge the children and parents who participated in the IDEFICS Project. Financial support: Part of the IDEFICS Project, this study is published on behalf of the IDEFICS European Consortium (idefics.eu). This project was supported by the European Community's Sixth RTD Framework Programme Contract No. 016181 (FOOD). The funder had no role in the design, analysis or writing of this article. Conflict of interest: None. Authorship: J.M.F.-A. carried out the statistical analyses with the help of C.B. and drafted the manuscript. K.B. supervised the quality control study protocol. A.H., G.E., T.V., Y.A.K., E.K. and L.A.M. supervised the national data collection procedures. All authors helped in data collection, read and critically reviewed the manuscript. Ethics of buman subject participation: The study was conducted according to the declaration of 
Helsinki. Each participating centre obtained ethical approval from its health research ethics authority. All children provided oral consent and their parents provided written informed consent for all examinations and for the collection of samples, subsequent analysis and storage of personal data and collected samples.

\section{Supplementary material}

To view supplementary material for this article, please visit https://doi.org/10.1017/S1368980017002361

\section{References}

1. Ng M, Fleming T, Robinson M et al. (2014) Global, regional, and national prevalence of overweight and obesity in children and adults during 1980-2013: a systematic analysis for the Global Burden of Disease Study 2013. Lancet 384, 766-781.

2. Stamatakis E, Wardle J \& Cole TJ (2010) Childhood obesity and overweight prevalence trends in England: evidence for growing socioeconomic disparities. Int J Obes (Lond) 34, 41-47.

3. Ogden CL, Carroll MD, Kit BK et al. (2014) Prevalence of childhood and adult obesity in the United States, 2011-2012. JAMA 311, 806-814.

4. Popkin BM, Adair LS \& Ng SW (2012) Global nutrition transition and the pandemic of obesity in developing countries. Nutr Rev 70, 3-21.

5. Ahrens W, Pigeot I, Pohlabeln H et al. (2014) Prevalence of overweight and obesity in European children below the age of 10. Int J Obes (Lond) 38, Suppl. 2, S99-S107.

6. Park MH, Falconer C, Viner RM et al. (2012) The impact of childhood obesity on morbidity and mortality in adulthood: a systematic review. Obes Rev 13, 985-1000.

7. Reilly JJ \& Kelly J (2011) Long-term impact of overweight and obesity in childhood and adolescence on morbidity and premature mortality in adulthood: systematic review. Int $J$ Obes (Lond) 35, 891-898.

8. $\mathrm{Hu}$ FB (2013) Resolved: there is sufficient scientific evidence that decreasing sugar-sweetened beverage consumption will reduce the prevalence of obesity and obesity-related diseases. Obes Rev 14, 606-619.

9. Rosenheck R (2008) Fast food consumption and increased caloric intake: a systematic review of a trajectory towards weight gain and obesity risk. Obes Rev 9, 535-547.

10. Hu FB (2002) Dietary pattern analysis: a new direction in nutritional epidemiology. Curr Opin Lipidol 13, 3-9.

11. Ambrosini GL (2014) Childhood dietary patterns and later obesity: a review of the evidence. Proc Nutr Soc 73, 137-146.

12. Fernandez-Alvira JM, Bornhorst C, Bammann K et al. (2015) Prospective associations between socio-economic status and dietary patterns in European children: the Identification and Prevention of Dietary- and Lifestyle-induced Health Effects in Children and Infants (IDEFICS) Study. Br J Nutr 113, 517-525.

13. Mikkila V, Rasanen L, Raitakari OT et al. (2007) Major dietary patterns and cardiovascular risk factors from childhood to adulthood. The Cardiovascular Risk in Young Finns study. Br J Nutr 98, 218-225.

14. Cutler GJ, Flood A, Hannan PJ et al. (2012) Association between major patterns of dietary intake and weight status in adolescents. Br J Nutr 108, 349-356.

15. Shroff MR, Perng W, Baylin A et al. (2014) Adherence to a snacking dietary pattern and soda intake are related to the development of adiposity: a prospective study in school-age children. Public Health Nutr 17, 1507-1513.
16. Oellingrath IM, Svendsen MV \& Brantsaeter AL (2011) Tracking of eating patterns and overweight - a follow-up study of Norwegian schoolchildren from middle childhood to early adolescence. Nutr J 10, 106.

17. Johnson L, Mander AP, Jones LR et al. (2008) Energy-dense, low-fiber, high-fat dietary pattern is associated with increased fatness in childhood. Am J Clin Nutr 87, 846-854.

18. Ambrosini GL, Emmett PM, Northstone $\mathrm{K}$ et al. (2012) Identification of a dietary pattern prospectively associated with increased adiposity during childhood and adolescence. Int J Obes (Lond) 36, 1299-1305.

19. Northstone K, Smith AD, Newby PK et al. (2013) Longitudinal comparisons of dietary patterns derived by cluster analysis in 7- to 13-year-old children. Br J Nutr 109, 2050-2058.

20. Ahrens W, Bammann K, Siani A et al. (2011) The IDEFICS cohort: design, characteristics and participation in the baseline survey. Int J Obes (Lond) 35, Suppl. 1, S3-S15.

21. Bammann K, Peplies J, Sjöström M et al. (2006) Assessment of diet. physical activity and biological, social and environmental factors in a multi-centre European project on diet- and lifestyle-related disorders in children (IDEFICS). J Public Health 14, 279-289.

22. Huybrechts I, Bornhorst C, Pala V et al. (2011) Evaluation of the Children's Eating Habits Questionnaire used in the IDEFICS study by relating urinary calcium and potassium to milk consumption frequencies among European children. Int J Obes (Lond) 35, Suppl. 1, S69-S78.

23. Lanfer A, Hebestreit A, Ahrens W et al. (2011) Reproducibility of food consumption frequencies derived from the Children's Eating Habits Questionnaire used in the IDEFICS study. Int J Obes (Lond) 35, Suppl. 1, S61-S68.

24. Freedman DS \& Sherry B (2009) The validity of BMI as an indicator of body fatness and risk among children. Pediatrics 124, Suppl. 1, S23-S34.

25. Javed A, Jumean M, Murad MH et al. (2015) Diagnostic performance of body mass index to identify obesity as defined by body adiposity in children and adolescents: a systematic review and meta-analysis. Pediatr Obes 10, 234-244.

26. Stomfai S, Ahrens W, Bammann K et al. (2011) Intra- and inter-observer reliability in anthropometric measurements in children. Int J Obes (Lond) 35, Suppl. 1, S45-S51.

27. Cole TJ \& Lobstein T (2012) Extended international (IOTF) body mass index cut-offs for thinness, overweight and obesity. Pediat Obes 7, 284-294.

28. United Nations Educational, Scientific and Cultural Organization (2006) International Standard Classification of Education: ISCED 1997. Montreal: UNESCO.

29. Organisation for Economic Co-operation and Development (2008) Growing Unequal? Income Distribution and Poverty in OECD Countries. Paris: OECD.

30. Bammann K, Gwozdz W, Lanfer A et al. (2013) Socioeconomic factors and childhood overweight in Europe: results from the multi-centre IDEFICS study. Pediatr Obes 8, 1-12.

31. Walthouwer MJ, Oenema A, Soetens K et al. (2014) Are clusters of dietary patterns and cluster membership stable over time? Results of a longitudinal cluster analysis study. Appetite 82, 154-159.

32. Northstone K \& Emmett PM (2008) Are dietary patterns stable throughout early and mid-childhood? A birth cohort study. Br J Nutr 100, 1069-1076.

33. Pala V, Lissner L, Hebestreit A et al. (2013) Dietary patterns and longitudinal change in body mass in European children: a follow-up study on the IDEFICS multicenter cohort. Eur J Clin Nutr 67, 1042-1049.

34. Smith AD, Emmett PM, Newby PK et al. (2014) Dietary patterns and changes in body composition in children between 9 and 11 years. Food Nutr Res 2014, 58.

35. Regber S, Novak M, Eiben G et al. (2013) Assessment of selection bias in a health survey of children and families the IDEFICS Sweden-study. BMC Public Health 13, 418. 